\title{
3D Collapse of Rotating Stellar Iron Cores in General Relativity Including Deleptonization and a Nuclear Equation of State
}

\author{
C. D. Ott, ${ }^{1,5, *}$ H. Dimmelmeier, ${ }^{2}$ A. Marek, ${ }^{2}$ H.-T. Janka, ${ }^{2}$ I. Hawke, ${ }^{3}$ B. Zink, ${ }^{2,4}$ and E. Schnetter ${ }^{4}$ \\ ${ }^{1}$ Max-Planck-Institut für Gravitationsphysik, Albert-Einstein-Institut, Am Mühlenberg 1, D-14476 Potsdam, Germany \\ ${ }^{2}$ Max-Planck-Institut für Astrophysik, Karl-Schwarzschild-Straße 1, D-85741 Garching, Germany \\ ${ }^{3}$ School of Mathematics, University of Southampton, Southampton SO17 1BJ, UK \\ ${ }^{4}$ Center for Computation and Technology, Louisiana State University, Baton Rouge, Louisiana 70803, USA \\ ${ }^{5}$ Steward Observatory and Department of Astronomy, University of Arizona, 933 N. Cherry Avenue, Tucson, AZ 85721, USA
}

(Received 29 September 2006; published 29 June 2007)

\begin{abstract}
We present 2D and 3D simulations of the collapse of rotating stellar iron cores in general relativity employing a nuclear equation of state and an approximate treatment of deleptonization. We compare fully general relativistic and conformally flat evolutions and find that the latter treatment is sufficiently accurate for the core-collapse supernova problem. We focus on gravitational wave $(\mathrm{GW})$ emission from rotating collapse, bounce, and early postbounce phases. Our results indicate that the GW signature of these phases is much more generic than previously estimated. We also track the growth of a nonaxisymmetric instability in one model, leading to strong narrow-band GW emission.
\end{abstract}

Introduction. - For more than two decades, astrophysicists have struggled to compute the gravitational wave (GW) signal produced by rotating stellar core collapse and the subsequent supernova evolution. Besides the coalescence of black hole and neutron star binaries, corecollapse events are considered to be among the most promising sources of detectable GWs. Theoretical predictions are still hampered by three major problems: (i) the rotational configuration prior to gravitational collapse is still uncertain since multi- $D$ evolutionary calculations of rotating massive stars have not yet been performed; (ii) reliable waveform estimates require a general relativistic (GR) treatment, since both high densities and velocities in combination with strong gravitational fields are encountered in this problem; and (iii) an adequate treatment of the nuclear equation of state (EOS) and the neutrino microphysics and radiative transfer is crucial for obtaining realistic collapse, bounce, and postbounce dynamics and waveforms. GW emission from core-collapse supernovae may arise from rotating collapse and bounce, postbounce neutrino-driven convection, anisotropic neutrino emission, nonaxisymmetric rotational instabilities of the protoneutron star (PNS), or from the recently proposed PNS core $g$-mode oscillations. Previous estimates of the GW signature of core-collapse supernovae have either relied on Newtonian simulations [1-6] (to some extent approximating GR effects [7]) or GR simulations with simplified analytic (so-called hybrid) EOSs and no neutrino treatment [8-11]. Depending on rotation, softness of the EOS at subnuclear densities, and inclusion of GR effects, the collapse dynamics and accordingly the GW signature can differ significantly.

Here, we present new results from GR simulations, focusing on the rotating collapse, bounce, and early postbounce phases. These are the first-ever multi- $D$ GR simu- lations with presupernova models from stellar evolution calculations, a microphysical nuclear EOS, and a simple but effective treatment of electron capture and neutrino radiation effects during collapse. In this way, we obtain the most accurate estimates of the $\mathrm{GW}$ signature of rotating stellar core collapse in full GR to date.

Method and initial models. - We perform all 3D simulations in $3+1$ GR using CACTUS [12], Cartesian coordinates, and mesh refinement provided by CARPET [13]. Spacetime is evolved using the BSSN formulation (see, e.g., [14]) with $1+\log$ slicing and a hyperbolic shift [15]. We use the finite-volume GR hydrodynamics code WHISKY [16]. Typical simulation grids extend to $3000 \mathrm{~km}$ and use 9 refinement levels. The central resolution is $\Delta r \sim 350 \mathrm{~m}$. In addition, we perform axisymmetric (2D) simulations for all models using the COCONUT code [8,17], approximating GR by the conformal flatness condition (CFC). COCONUT utilizes spherical coordinates with 250 logarithmicallyspaced radial zones with $\Delta r \sim 250 \mathrm{~m}$ and 45 equidistant angular zones. Resolution tests with both codes do not yield significant qualitative or quantitative differences. We extract GWs using a variant of the Newtonian quadrupole formula [9].

We employ the microphysical nuclear EOS of Shen et al. [18] as implemented in [19]. Deleptonization is approximated as proposed by [20]: The electron fraction $Y_{e}$ is parameterized as a function of density based on data from spherically symmetric radiation-hydrodynamics calculations with standard electron capture rates [21]. After core bounce, $Y_{e}$ is passively advected, and further lepton loss is neglected, but neutrino pressure continues to be taken into account above trapping density [20].

In this Letter, we focus on the collapse of massive presupernova iron cores with at most moderate differential rotation, and rotation rates that may be too fast to match 
TABLE I. Summary. $\rho_{b}$ is the density at bounce, the maximum characteristic GW strain $h_{\text {char,max }}$ is at a distance of $10 \mathrm{kpc}$, and $E_{\mathrm{gw}}$ is the energy emitted in GWs. Values for E20A $\mathrm{pb}$ include GW emission from late-time 3D dynamics.

\begin{tabular}{lcccccc}
\hline \hline Model & $A\left[10^{8} \mathrm{~cm}\right]$ & $\beta_{i}[\%]$ & $\beta_{b}[\%]$ & $\rho_{b}\left[\frac{10^{14} \mathrm{~g}}{\mathrm{~cm}^{3}}\right]$ & $h_{\text {char, } \max }\left[10^{-21}\right]$ & $E_{\mathrm{gw}}\left[10^{-9} M_{\odot} c^{2}\right]$ \\
\hline s20A1B1 & 50.0 & 0.25 & 0.90 & 3.29 & 1.46 & 0.6 \\
s20A1B5 & 50.0 & 4.00 & 10.52 & 2.90 & 9.68 & 26.9 \\
s20A2B2 & 1.0 & 0.50 & 6.72 & 3.07 & 8.77 & 22.0 \\
s20A2B4 & 1.0 & 1.80 & 16.33 & 2.35 & 4.28 & 9.4 \\
s20A3B3 & 0.5 & 0.90 & 16.57 & 2.33 & 4.58 & 12.4 \\
E20A & $\cdots$ & 0.37 & 11.31 & 2.79 & 12.18 & 36.9 \\
E20A $_{\mathrm{pb}}$ & & & & 24.23 & 75.4 \\
\hline \hline
\end{tabular}

garden-variety pulsar birth spin estimates [22,23], but could be relevant in the collapsar-type gamma-ray burst context. As initial data, we use the nonrotating $20 M_{\odot}$ presupernova model s20 of Woosley et al. [24], which we force to rotate according to the rotation law discussed in $[5,8]$. We parameterize our models in terms of the differential rotation parameter $A$ and the initial ratio of rotational kinetic to gravitational energy $\beta_{i}=T /|W|$. In addition, we perform a calculation with the $20 M_{\odot}$ model E20A of [25], which includes an approximate 1D treatment of rotation. In Table I, we list the model parameters.

Results. - In Fig. 1, we compare GW signals computed with COCONUT in 2D-CFC and those computed with our 3D-full-GR approach. Model s20A2B2 (red lines) is a moderate rotator with a $\beta_{i}=0.50 \%$, rotating rigidly in its central region. It stays axisymmetric throughout its numerical evolution. The agreement of $2 \mathrm{D}-\mathrm{CFC}$ with $3 \mathrm{D}-$ full-GR is excellent for this model: Both waveforms match almost perfectly at bounce and during the very early postbounce phase. A few ms after bounce, when convection in the region behind the stalling shock sets in due to a negative entropy gradient, the signals begin to differ quantitatively while remaining in phase. We attribute this small mismatch to the choice of coordinate grids and to differences in the growth and scale of vortical postbounce motions between 2D and 3D. Model s20A1B5 rotates with

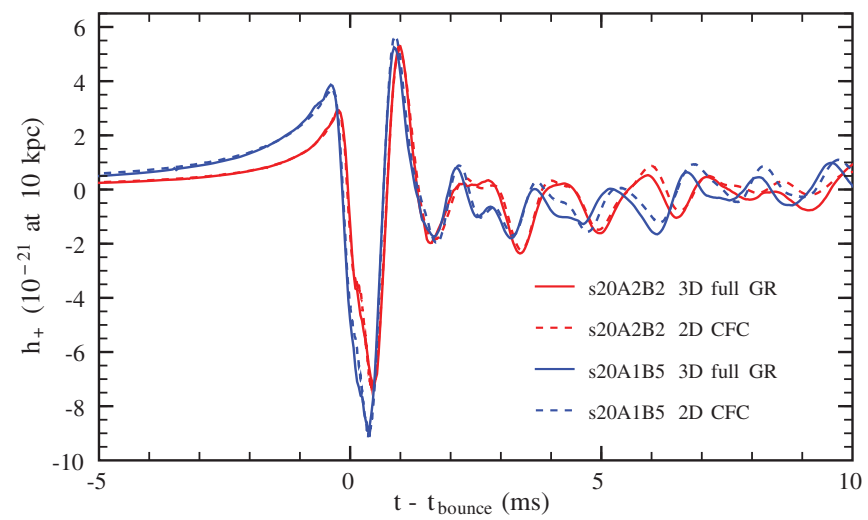

FIG. 1 (color). GW strain $h_{+}$along the equator for models s20A2B2 and s20A1B5. We compare 2D-CFC and 3D-full-GR results. constant $\Omega$ in the entire core. Despite its very large $\beta_{i}=$ $4 \%$, it remains essentially axisymmetric during the time covered by our simulation, since most of its angular momentum is attached to material at large radii that falls inward and spins up only slowly. The waveforms in CFC and full GR agree very well. Again, both waveforms match best for the strong burst related to core bounce during which more than $\sim 90 \%$ of the total GW energy are emitted in an axisymmetric model. The overall excellent agreement of CFC with full GR confirms results of $[9,11]$ and proves that CFC is a very good approximation to full GR in the core-collapse scenario.

In Fig. 2, we present waveforms of models with varying initial degree of differential rotation $A$ and rotation rate $\beta_{i}$. We find that the inclusion of a microphysical EOS and of electron capture yields results that differ considerably from those obtained in previous, less sophisticated studies. Figure 2 exemplifies that largely independent of the initial rotational configuration, the $\mathrm{GW}$ signal of rotating collapse has a generic shape: a slow rise in the prebounce phase, a large negative amplitude at bounce when the motion of the inner core is reversed, followed by a ring-down. This socalled "Type I" signature corresponds to a baryonic pressure dominated bounce $[1,2,5,8]$. Thus, all our models undergo core bounce dominated by the stiffening of the EOS at nuclear density.

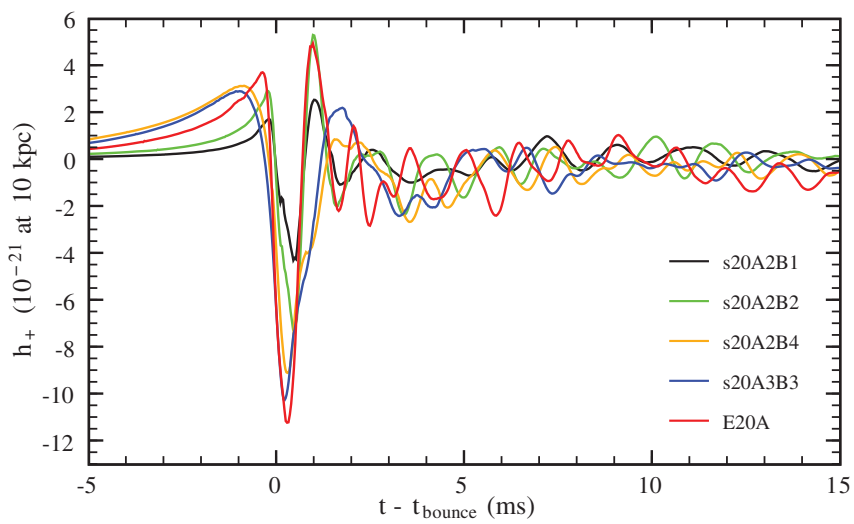

FIG. 2 (color). Equatorial GW strain $h_{+}$for a representative subset of the models listed in Table I. Note the generic shape of the core bounce GW signal. 
This is in stark contrast to the studies using the simple hybrid EOS [2,8-10], where initial models with rotation rates in the range investigated here develop sufficient centrifugal support during contraction to stop collapse at subnuclear densities, resulting in several consecutive centrifugal bounces separated by phases of coherent reexpansion of the inner core. While in GR, models exhibiting such multiple centrifugal bounce and the corresponding "Type II" GW signals are only rarer compared to Newtonian gravity [8], we do not observe any such model in this study. An evident example is model s20A2B4: In previous studies without detailed microphysics, the corresponding model with identical initial rotation parameters (A2B4G1) showed clear "Type II" behavior in both Newtonian and GR calculations $[2,8]$.

The suppression of the multiple centrifugal bounce scenario is due to two physical effects: (i) In contrast to the simple hybrid EOS, in our case the mass and dynamics of the inner core (which is most important for the GW emission) is not merely determined by the adiabatic index $\gamma=$ $d \ln P / d \ln \rho$ (at constant entropy) of the EOS, but also by deleptonization during collapse. This leads to considerably smaller inner cores with less angular momentum and weaker pressure support. (ii) Since multiple centrifugal bounce was observed for a model with moderately fast rotation in a previous Newtonian study [1] employing both a microphysical EOS and deleptonization, the absence of this collapse type in our study is not only caused by microphysical effects, but also by the effectively stronger gravity in GR. This is in accordance with simulations using the simple hybrid EOS [8]. For a more detailed discussion of these effects, see [26].

Model E20A possesses the largest GW amplitude of all our models. In addition, it reaches a high $\beta_{b}$ at core bounce (see Table I) and settles at a postbounce $\beta_{f}$ of $\sim 9 \%$. A previous Newtonian study [27] has found a low- $T /|W|$ nonaxisymmetric instability for a PNS with similar $\beta_{f}$. In order to verify their findings, we trace the evolution of model E20A to $70 \mathrm{~ms}$ after bounce and perform an analysis of azimuthal density modes $\propto e^{i m \varphi}$ by computing complex Fourier amplitudes $C_{m}=\frac{1}{2 \pi} \int_{0}^{2 \pi} \rho(\varpi, \varphi, z=0) e^{i m \varphi} d \varphi$ on rings of constant coordinate radius with respect to the coordinate center of mass. The latter stays within the innermost zone at all times. In the top panel of Fig. 3, we display the normalized mode amplitudes extracted at $15 \mathrm{~km}$ radius. Without adding artificial seed perturbations to model E20A, discretization errors trigger $m=\{1,2,3\}$ modes, which rise to a level of $\sim 10^{-5}$ during the $\sim 220 \mathrm{~ms}$ collapse phase. After bounce, the $m=1$ mode exhibits the fastest growth. This growth on a dynamical time scale, lasting over tens of ms until saturation, is closely followed by a growth of $m=\{2,3\}$ daughter modes [27,28]. Note that after core bounce, model E20A remains dynamically stable to the $m=4$ grid mode. In the lower panel of Fig. 3, we plot the GW strains $h_{+}$and $h_{\times}$as seen along the polar axis. The rotational symmetry of E20A before and shortly

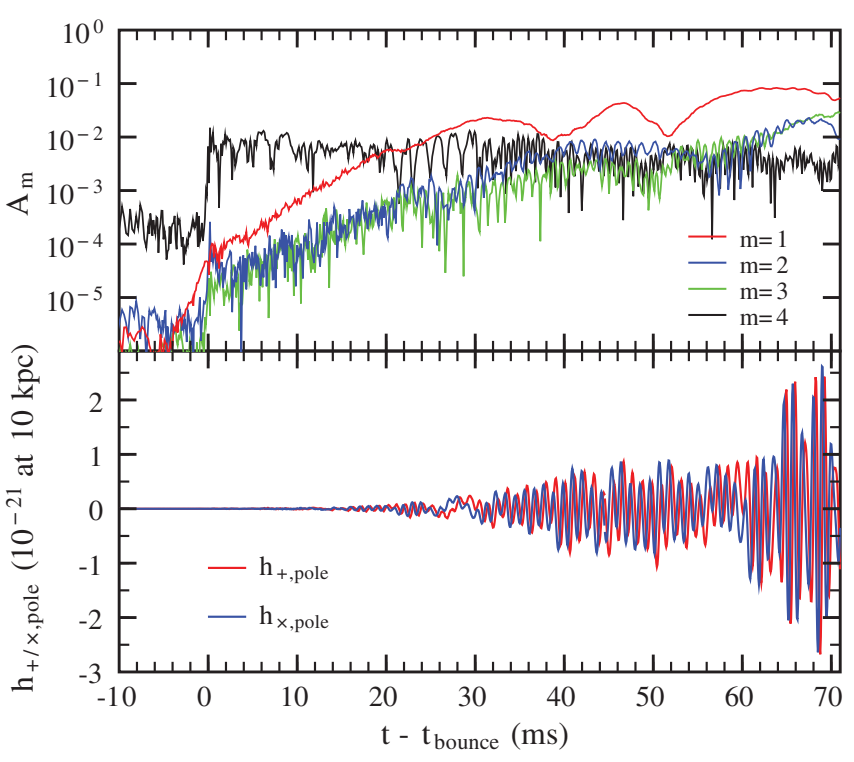

FIG. 3 (color). Normalized mode amplitudes $A_{m}=\left|C_{m}\right| / C_{0}$ at postbounce times (upper panel) and GW strains $h_{+}$and $h_{\times}$ along the poles (lower panel) for model E20A.

after bounce is reflected in the fact that $h_{+}$and $h_{\times}$are practically zero until E20A develops considerable nonaxisymmetry when the $m=1$ mode becomes dominant and its $m=2$, GW-emitting harmonic reaches a sizable amplitude. In agreement with expectations for a spinning bar, $h_{+}$ and $h_{\times}$oscillate at the same frequency $(\sim 930 \mathrm{~Hz})$ and are phase-shifted by a quarter cycle.

Discussion. - Our results indicate that the GW signature of the collapse, bounce, and early postbounce phases of the core-collapse supernova evolution is much more generic than previously thought. We find that the dynamics of core bounce are dominated by mainly gravity and microphysics, reducing the importance of centrifugal support for the rotation rates considered here. Importantly, for our model set, we do not observe rotationally induced multiple core bounce at subnuclear density as proposed by previous studies that did not include a microphysical EOS and electron capture treatment in combination with GR.

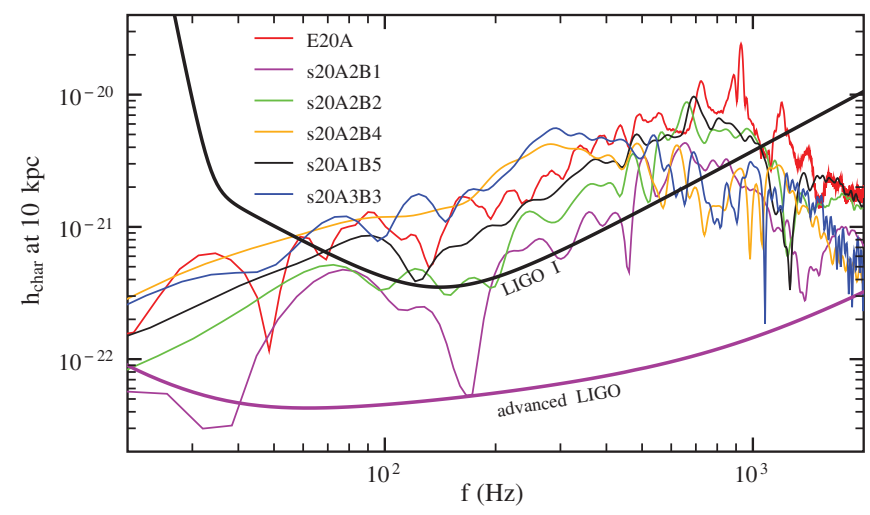

FIG. 4 (color). Spectra of the characteristic GW strain $h_{\text {char }}$ of all models and the LIGO (optimal) rms noise curves [32]. 
Thus, we predict that the core-bounce waveform of models in a large parameter space of initial rotation rate and degree of differential rotation will likely both qualitatively and quantitatively resemble those presented in Fig. 2.

Model E20A, which we evolve to later postbounce times, exhibits the dynamical growth of a nonaxisymmetric low- $T /|W|$ corotation-type $m=1$ instability [27-29]. We also find $m=\{2,3\}$ contributions and significant $\mathrm{GW}$ emission from the quadrupole components. We emphasize that we observe this instability not only in E20A, but also in other models with comparable values of $\beta_{f}$. Our results, which remove the limitations of previous studies $[10,27,30,31]$, demonstrate that the development of nonaxisymmetric structures is neither limited to Newtonian gravity, simple matter models, equilibrium configurations, nor high values of $\beta$, but may rather be a phenomenon occurring generically in differentially rotating compact stars.

For the GW signals from the axisymmetric collapse and core-bounce phase, we obtain peak amplitudes of up to $h \sim$ $10^{-20}$ at $10 \mathrm{kpc}$, while the nonaxisymmetric structures in model E20A developing later emit GWs with $h$ only a factor $\sim 5$ smaller. However, since the latter emission process operates over several tens of $\mathrm{ms}$, the total energy $E_{\mathrm{gw}}$ emitted in GWs is larger than that from the core-bounce signal. This is evident in Fig. 4, where we display the characteristic GW strain spectra $h_{\text {char }}=$ $R^{-1} \sqrt{2 \pi^{-2} d E_{\mathrm{gw}} / d f}$ [5] for all models, evolving E20A for $70 \mathrm{~ms}$ after bounce (see also Table I). Considering only the core-bounce waveforms, $h_{\text {char }}$ has its maximum between 300 and $800 \mathrm{~Hz}$, while for model E20A it peaks at $\sim 930 \mathrm{~Hz}$, the frequency of the GW-emitting component of its nonaxisymmetric structures. We conclude that the corebounce GW signals of all models investigated here may be detectable by current and future LIGO-class detectors from anywhere in the Milky Way. Models that develop nonaxisymmetric instabilities may be detectable out to much larger distances if the instability persists for a sufficiently long time.

We point out that due to the nature of the approximation used for the neutrino effects, we can only accurately model the GW emission in the collapse, bounce, and early postbounce epoch of the core-collapse supernova scenario. In future work, we plan to improve upon this and carry out longer-term postbounce evolutions, where other GW emission mechanisms may be relevant $[6,7]$.

We thank A. Burrows, E. Müller, S. Ou, L. Rezzolla, E. Seidel, D. Shoemaker, N. Stergioulas, and J. Tohline for help and stimulating discussions. This research was partially supported by the DFG (Nos. SFB/TR7, SFB 375) and by the NCSA (Grant No. AST050022N).

\footnotetext{
*cott@as.arizona.edu
}

[1] R. Mönchmeyer, G. Schäfer, E. Müller, and R. Kates, Astron. Astrophys. 246, 417 (1991).

[2] T. Zwerger and E. Müller, Astron. Astrophys. 320, 209 (1997).

[3] M. Rampp, E. Müller, and M. Ruffert, Astron. Astrophys. 332, 969 (1998).

[4] K. Kotake, S. Yamada, K. Sato, K. Sumiyoshi, H. Ono, and H. Suzuki, Phys. Rev. D 69, 124004 (2004).

[5] C.D. Ott, A. Burrows, E. Livne, and R. Walder, Astrophys. J. 600, 834 (2004).

[6] C. D. Ott, A. Burrows, L. Dessart, and E. Livne, Phys. Rev. Lett. 96, 201102 (2006).

[7] E. Müller, M. Rampp, R. Buras, H.-T. Janka, and D. H. Shoemaker, Astrophys. J. 603, 221 (2004).

[8] H. Dimmelmeier, J.A. Font, and E. Müller, Astron. Astrophys. 388, 917 (2002); 393, 523 (2002).

[9] M. Shibata and Y.-I. Sekiguchi, Phys. Rev. D 69, 084024 (2004).

[10] M. Shibata and Y.-I. Sekiguchi, Phys. Rev. D 71, 024014 (2005).

[11] P. Cerdá-Durán, G. Faye, H. Dimmelmeier, J. A. Font, J.M. Ibáñez, E. Müller, and G. Schäfer, Astron. Astrophys. 439, 1033 (2005).

[12] http://www.cactuscode.org.

[13] E. Schnetter, S.H. Hawley, and I. Hawke, Classical Quantum Gravity 21, 1465 (2004).

[14] T. W. Baumgarte and S. L. Shapiro, Phys. Rep. 376, 41 (2003).

[15] M. Shibata, Astrophys. J. 595, 992 (2003).

[16] L. Baiotti, I. Hawke, P. J. Montero, F. Löffler, L. Rezzolla, N. Stergioulas, J. A. Font, and E. Seidel, Phys. Rev. D 71, 024035 (2005).

[17] H. Dimmelmeier, J. Novak, J. A. Font, J. M. Ibáñez, and E. Müller, Phys. Rev. D 71, 064023 (2005).

[18] H. Shen, H. Toki, K. Oyamatsu, and K. Sumiyoshi, Prog. Theor. Phys. 100, 1013 (1998).

[19] A. Marek, H.-T. Janka, R. Buras, M. Liebendörfer, and M. Rampp, Astron. Astrophys. 443, 201 (2005).

[20] M. Liebendörfer, Astrophys. J. 633, 1042 (2005).

[21] R. Buras, H.-T. Janka, M. Rampp, and K. Kifonidis, Astron. Astrophys. 457, 281 (2006).

[22] A. Heger, S. E. Woosley, and H. C. Spruit, Astrophys. J. 626, 350 (2005).

[23] C. D. Ott, A. Burrows, T. A. Thompson, E. Livne, and R. Walder, Astrophys. J. Suppl. Ser. 164, 130 (2006).

[24] S.E. Woosley, A. Heger, and T. A. Weaver, Rev. Mod. Phys. 74, 1015 (2002).

[25] A. Heger, N. Langer, and S. E. Woosley, Astrophys. J. 528, 368 (2000).

[26] H. Dimmelmeier, C. D. Ott, H.-T. Janka, A. Marek, and E. Müller, Phys. Rev. Lett. (to be published).

[27] C. D. Ott, S. Ou, J. E. Tohline, and A. Burrows, Astrophys. J. Lett. 625, L119 (2005).

[28] S. Ou and J. E. Tohline, Astrophys. J. 651, 1068 (2006).

[29] M. Saijo and S. Yoshida, Mon. Not. R. Astron. Soc. 368, 1429 (2006).

[30] J. M. Centrella, K. C. B. New, L. L. Lowe, and J. D. Brown, Astrophys. J. Lett. 550, L193 (2001).

[31] M. Shibata, S. Karino, and Y. Eriguchi, Mon. Not. R. Astron. Soc. 334, L27 (2002).

[32] D. H. Shoemaker (private communication). 